\title{
O.S.P.
}

L'orientation scolaire et professionnelle

$29 / 1 \mid 2000$

Le conseil en orientation

\section{Le conseil en orientation : introduction}

Career counseling : introduction

Serge Blanchard

\section{(2) OpenEdition}

Journals

Édition électronique

URL : http://journals.openedition.org/osp/12827

DOI : $10.4000 /$ osp. 12827

ISSN : 2104-3795

Éditeur

Institut national d'étude du travail et d'orientation professionnelle (INETOP)

Édition imprimée

Date de publication : 15 mars 2000

Pagination : $3-26$

ISSN : 0249-6739

\section{Référence électronique}

Serge Blanchard, «Le conseil en orientation : introduction », L'orientation scolaire et professionnelle [En ligne], 29/1 | 2000, mis en ligne le 15 mars 2002, consulté le 12 novembre 2020. URL : http:// journals.openedition.org/osp/12827 ; DOI : https://doi.org/10.4000/osp.12827

Ce document a été généré automatiquement le 12 novembre 2020.

(C) Tous droits réservés 


\title{
Le conseil en orientation : introduction
}

\author{
Career counseling : introduction
}

Serge Blanchard

1 Au cours de ces vingt dernières années, les institutions en charge de l'orientation scolaire et professionnelle se sont différenciées et le nombre de conseillers a beaucoup augmenté. Actuellement, les activités de conseil en orientation occupent environ 10000 conseillers, qui reçoivent surtout des jeunes (public scolaire et jeunes de moins de 25 ans) dans les centres d'information et d'orientation (C.I.O.), les missions locales (M.L.) et les permanences $d$ ' accueil pour l'information et l'orientation (P.A.I.O.), et environ 20000 conseillers, qui reçoivent surtout des jeunes sortis du système scolaire et des adultes dans les agences locales de l'agence nationale pour l'emploi (A.N.P.E.), dans les centres de l'association pour la formation professionnelle des adultes (A.F.P.A.) et dans les Centres Interinstitutionnels de Bilan de Compétences (C.I.B.C.). Si l'on ajoute à ces structures, les services de conseil dispensés par des personnels travaillant dans des associations, comme par exemple Retravailler (Sullerot, 1996; Pegeault \& BerSchiavetta, 1997), ou dans des services existant au sein même d'entreprises publiques (Hurbin, 1997), ou au sein d'entreprises privées (Loss \& Parlier, 1996), ce sont plus de 30000 personnes qui exercent des fonctions de conseil en orientation en France.

2 On sait par ailleurs que le conseil s'est étendu à de nombreux domaines, comme ceux du conseil conjugal (Lemaire, 1986), de l'animation de divers groupes d'aide à des personnes qui cherchent, par exemple, à perdre du poids ou à arrêter de fumer, de la psychologie de la santé (Tourette-Turgis, 1996), de l'aide téléphonique aux candidats au suicide (Lancry, 2000), etc. De plus, de nombreux professionnels dont ce n'est pas la fonction principale (médecins généralistes, infirmières, assistantes sociales, etc.) ont fréquemment l'occasion d'avoir une activité de conseil. Si c'est essentiellement la question du conseil en orientation qui est traitée dans ce numéro, bien des questions qui y sont abordées concernent aussi d'autres champs du conseil, d'autant que le courant nord-américain du counseling tend à élargir le conseil en orientation au 
conseil personnel et même à la thérapie. Les questions abordées dans ce numéro intéressent donc un large public.

3 Après avoir présenté les principaux thèmes de réflexion qui sont développés dans les articles de ce numéro, nous aborderons la question des relations entre les théories et les pratiques du conseil en orientation.

\section{Quelques réflexions et propositions sur les théories et les pratiques du conseil en orientation}

Dans ce numéro consacré au thème du conseil en orientation, nous avons choisi de présenter des points de vue divers: cinq articles d'auteurs français, deux articles d'auteurs anglophones (une anglaise, trois américains des Etats-Unis) et un article d'un québécois et d'un français. Notons toutefois que tous situent leur réflexion dans le champ de la psychologie.

5 En premier lieu, il paraît important de s'arrêter sur la définition du mot conseil. L'activité de conseil en orientation consiste-t-elle uniquement à donner des conseils ? Rappelons que le mot conseil renvoie à deux sens principaux, comme l'atteste la définition suivante extraite du Dictionnaire historique de la langue française d'A. Rey (1995, p. 477).

6 CONSEIL, nom masculin, est issu (v. 980) du latin consilium d'abord employé dans la langue juridique pour "endroit où l'on délibère" par métonymie "consultation, délibération", et passé dans la langue commune au sens de "projet, dessein » et notamment «dessein mûri et réfléchi » d'où «bon avis, sagesse, prévoyance ». Consilium est dérivé de consulere, « réunir pour une délibération » (consulter).

7 Le mot apparaît avec le sens de " avis que l'on donne à qqn sur ce qu'il doit faire ", dont participe la locution proverbiale la nuit porte conseil (1611) ... Un autre emploi métonymique, « personne ne qui porte conseil, qui donne des conseils» (fin XIIe s.) a disparu au profit de conseiller, mais s'est maintenu dans certaines professions juridiques avec la valeur de « personne qui en assiste une autre dans la direction de ses affaires » (conseil fiscal et dans des titres, ingénieur-conseil) : cf. consultant. Le sens de " réunion de gens qui délibèrent, délibération » (1080) connaît depuis l'ancien français une grande vitalité dans la dénomination d'institutions, héritées pour certaines de l'Ancien Régime (conseil d'Etat, 1790), apparus plus récemment pour d'autres : conseil général (1871), conseil régional (1972), propres à l'usage du français en France, ou conseil de l'Europe (1979).

8 Or, lorsqu'on évoque le mot conseil, c'est plus souvent l'acception « donner un conseil » qui vient généralement à l'esprit ainsi que les maximes et les proverbes qui en dénient l'utilité [par exemple: Il est toujours stupide de donner des conseils, mais en donner de bons est absolument fatal. (Oscar Wilde, Portrait of Mr. W. H.)]. Pourquoi se méfie-t-on généralement des conseils? Shaftesbury (1710/1993), philosophe anglais (1671-1713), propose une explication dans ses Exercices :

9 «En bien des occasions, j'ai entendu dire par des gens de bon sens que "En fait de conduite privée, un CONSEIL n'a jamais amélioré personne" et j'ai souvent pensé quelle mauvaise maxime c'est là. Mais à examiner la chose davantage, j'ai conclu qu'on pouvait accepter cette maxime sans porter grand tort au genre humain. Car si l'on songe à la manière dont un conseil est généralement donné, il n’y a pas de raison, je 
pense, de s'étonner qu'il soit si mal reçu. Il y a là quelque chose qui renverse étrangement la situation et fait que celui qui donne est le seul à y gagner. Car, d'après ce que j'ai pu observer maintes fois dans la vie, ce que nous appelons donner un conseil, c'est proprement saisir une occasion de montrer notre propre sagesse aux dépens d'autrui. De l'autre côté, se faire instruire, ou recevoir un conseil dans les conditions qui nous sont habituellement imposées, ce n'est guère mieux que de docilement donner à autrui l'occasion de se faire une qualité de nos défauts.

En réalité, aussi capable ou désireux de conseiller qu'un homme puisse être, ce n'est pas chose aisée que de faire d'un CONSEIL un don gratuit. Car pour qu'un don soit vraiment gratuit, il ne doit rien s'y trouver qui prenne à autrui pour nous apporter à nousmêmes. A tous autres égards, donner et dispenser, c'est générosité et bienveillance ; mais prodiguer de la sagesse, c'est se donner une allure de Maître qu'on ne nous permet pas si facilement. Les hommes apprennent volontiers toute autre chose qu'on leur enseigne. Ils peuvent supporter un Maître de mathématiques, de musique, ou de n'importe quelle autre science ; mais pas d'entendement et de bon sens ».

C'est à définir précisément le sens du tenir conseil qu'Alexandre Lhotellier s'attache dans son article. Tenir conseil, selon lui, doit être entendu comme une délibération en vue d'agir, démarche impliquant :

- la création d'une relation dialogique ;

- la construction méthodique et plurielle du sens d'une situation-problème,

- un travail sur le temps, c'est à dire une prise en compte du moment et du rythme ;

en vue d'élaborer une décision fondatrice d'une action sensée, responsable et autonomisante.

Selon sa conception, tenir conseil ne vise pas seulement une intention, un choix à faire ou une décision à prendre, mais aussi, fondamentalement, à réaliser un acte. Cet agir en situation d'une personne en devenir demande, pour qu'il prenne sens :

- une auto-réflexion critique, et les discours produits ne sont pas tous des rationalisations sommaires, des illusions, des idéologies fausses ;

- une capacité d'action délibérée.

14 La pratique du tenir conseil doit être régulée par une évaluation et il serait particulièrement important de savoir quelles représentations s'en font les usagers des institutions de conseil en tous genres. Le tenir conseil renvoie finalement à une dimension éthique : si l'on veut que le savoir ne se réduise pas à une consommation futile, mais au contraire se développe dans un vrai travail de soi, il y a bien nécessité de tenir conseil.

Certaines des idées développées par Alexandre Lhotellier à propos du tenir conseil semblent assez proches de la philosophie stoïcienne de Marc Aurèle, dont les règles de pensée et d'action invitent à "vivre en pleine conscience, en pleine lucidité, donner toute son intensité à chacun de ses instants et un sens à sa vie tout entière " (Hadot, 1997, p. 333). La démarche du tenir conseil paraît donc étroitement liée à une pensée qui relève de la psychologie philosophique. «Pour Paul Ricœur, il y a une psychologie philosophique qui n'est pas de l'ordre de la connaissance qui, elle, est scientifique, mais de l'ordre d'une pensée soit déductive, soit réflexive. Elle se pose sur l'homme trois

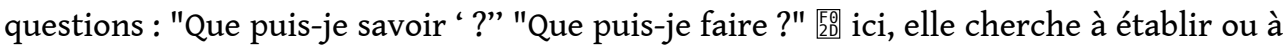
justifier une éthique 㜯 et enfin "Que puis-je espérer?" Question à laquelle ne peut 
répondre qu'une prospective où prédomine la recherche du sens de notre vie " (Fraisse, 1985, p. 335).

16 Cette démarche du tenir conseil s'appuie également sur les savoirs locaux limités (opposés aux savoirs sans limites fournis par les systèmes qui proposent une explication globalisante du monde et qu'on appelle mythes) de la psychologie scientifique, savoirs non disponibles à l'époque de Marc Aurèle, par exemple des savoirs sur les aspects émotionnels à prendre en compte au cours de la conduite de l'entretien de conseil, un thème que l'article suivant aborde.

Dans son article, Pascal Mallet se demande ce que la théorie de l'attachement peut apporter aux psychologues du conseil. Il peut paraître étrange de faire appel à une théorie de ce type, connue pour décrire la formation des premiers liens entre le bébé et sa mère, pour rendre compte des relations psychologiques entre un conseiller et un consultant (parfois même d'âge adulte). Après avoir passé en revue l'évolution des recherches concernant la théorie de l'attachement, Pascal Mallet envisage deux thèmes concernant le conseil en orientation

18 Le premier thème consiste à se demander s'il y a des liens entre les attachements confiants aux parents et la formation chez les adolescents d'intentions d'avenir scolaire et professionnel. Les adolescents soutenus par des attachements confiants à leurs parents se disent davantage enclins à poursuivre leur formation et la planifient plus activement. L'attachement confiant aux parents aurait un effet positif sur l'exploration des perspectives de formation scolaire et professionnelle, il soutiendrait l'adolescent dans les prises de risque requises par certaines orientations professionnelles. D'une façon plus générale, les attachements confiants aux parents apportent un soutien à l'affirmation de la personnalité des adolescents.

Dans la réalité, s'orienter est autant une affaire de deuil et de renoncement que de choix et de projet (Dumora, 1977). Face au découragement possible du consultant, une évaluation des soutiens socio-émotionnels dont il dispose, de ceux qu'il pourrait mobiliser, des moyens pour y parvenir, ne paraît pas relever d'une psychologisation excessive. De tels effets positifs du soutien socio-émotion nel ont été montrés à propos de la lutte contre des maladies graves et contre les conséquences de la perte d'emploi.

On a décrit des styles de personnalité sociale qui s'appliquent aux adolescents et on distingue notamment: ceux qui disposent d'attachements confiants; les anxieuxambivalents affectés par un doute obsédant; les anxieux-évitants qui ont tendance à ignorer les avis de leurs partenaires d'attachement ou à les éviter. Le conseiller pourrait moduler son comportement au cours de l'entretien en fonction des styles de ses consultants.

21 Le second thème abordé par Pascal Mallet consiste précisément à se demander en quoi la théorie de l'attachement peut aider à décrire les aspects socio-émotionnels en œuvre dans la conduite de l'entretien et dans l'examen de la situation du consultant. Selon Bolwby, les implications de la théorie de l'attachement à la conduite de l'entretien consistent notamment, pour le psychologue, à rechercher à établir une base sûre, une base de réconfort, à explorer les attachements passés et les difficultés relationnelles actuelles et à explorer la relation entre le consultant et le psychologue. Ainsi, avec les sujets anxieux, particulièrement sensibles aux confrontations, le conseiller doit bien expliciter ses sentiments sur ce qui se passe au cours de l'entretien afin qu'ils ne risquent pas de percevoir des critiques là où il n'y en a pas. 
dans la pratique. Nous avons déjà souligné que les savoirs scientifiques sont des savoirs
locaux et l'on doit toujours se poser la question de leur généralisabilité et de leur applicabilité aux champs plus larges de pratiques professionnelles. De plus, il semblerait que beaucoup de conseillers ne croient pas à l'utilité pratique des théories psychologiques, comme l'a déjà mis en évidence une étude anglaise publiée dans notre revue (Kidd et al., 1995). Selon Alison Fielding, si l'utilité du discours théorique est mal perçue par les praticiens du conseil, c'est en partie parce que les modèles théoriques traditionnels répondent mal aux besoins actuels des conseillers. Il est important de prendre en compte les évolutions actuelles qui devraient infléchir les conceptions traditionnelles de l'orientation professionnelle : changements dans les profils d'emploi, dans les façons d'envisager la vie professionnelle, utilisation des technologies de l'information, développement de l'apprentissage tout au long de la vie et, enfin, multiculturalisme. Alison Fielding propose un modèle d'orientation continue qui prend en compte le fait que, dans nos sociétés, les apprentissages se dérouleront tout au long de la vie. Ce modèle repose à la fois sur la psychologie et sur la sociologie et intègre donc à la fois des facteurs internes (différences individuelles, culture et style de vie) et des facteurs externes (développement du travail flexible, accessibilité des emplois, caractéristiques de l'environnement comme l'offre de formation, les attentes de son milieu familial...). Ce modèle n'implique pas une quelconque progression linéaire entre des étapes fixées d'avance. Il est ouvert et souple et peut être utilisé à tout moment pour aider le consultant et le praticien à déterminer la base de départ de la procédure de conseil, à se mettre d'accord sur les moyens à mettre en œuvre, afin de satisfaire au mieux les besoins manifestés. L'utilisation de ce modèle est illustrée par la présentation d'un cas. Dans sa conclusion, Alison Fielding s'interroge sur les relations entre théorie et pratique : les praticiens sont-ils plutôt des déchiffreurs de cartes, des cartographes ou des joueurs de jazz capables d'improviser sur un thème? Selon elle, l'essentiel, dans le conseil en orientation, c'est la compréhension en commun de ce qui doit être réalisé et de la façon de le réaliser (considérations proches de la notion d'alliance de travail à instaurer entre le consultant et le conseiller), en réinvestissant la théorie dans l'activité du praticien.

Dans leur article, Norman Gysbers, Mary Heppner et Joseph Johnston présentent une conception du conseil liée au développement de carrière tout au long de la vie. Pour eux, la différenciation opérée entre une forme de conseil axée sur le domaine personnel et émotionnel et une autre axée sur la carrière, est artificielle. En effet, dans la pratique, de nombreux consultants («clients» aux Etats-Unis) doivent faire face simultanément à des problèmes personnels et émotionnels et à des problèmes de carrière qui sont le plus souvent intriqués. Pour différencier les interventions 
conduites dans le cadre du vaste champ de l'aide au développement de carrière, les auteurs distinguent :

- le conseil à la carrière, défini comme une interaction de face à face prolongée entre le conseiller et son consultant, essentiellement focalisée sur les questions de travail et de carrière ; cette interaction est de nature psychologique et la relation entre le conseiller et le consultant jouent un rôle important ;

- la guidance de carrière, qui implique toutes les composantes 㙺 en termes de services et d'activités 疑 des institutions éducatives, agences et autre organismes qui offrent du conseil et des programmes de formation en rapport avec la carrière ;

- l'éducation à la carrière, qui tend à privilégier le processus d'enseignement/ apprentissage en tant que mode d'intervention principal du développement de carrière ; les professeurs et les éducateurs apparaissent comme les acteurs principaux de cette stratégie d'intervention.

Norman Gysbers, Mary Heppner et Joseph Johnston définissent leur modèle de «développement de carrière tout au long de la vie » comme un modèle qui Intègre la dimension de croissance et de développement de l'individu, considéré dans sa globalité, et qui prend en compte toutes les facettes de l'identité individuelle. Ce développement s'accompagne d'un processus continu d'interaction, et d'intégration des rôles, des cadres et des événements de la vie de l'individu, qui sont eux-mêmes influencés par des variables comme le genre, l'origine ethnique, la religion et le statut socio-économique.

L'un des objectifs principaux de la démarche de conseil de développement de carrière tout au long de la vie est d'amener le consultant à identifier, décrire et comprendre ce développement afin de faire émerger une conscience de carrière, qui repose sur la notion de sois possibles de Markus et Nurius (Kilhs trom et al., 1988/1992, pp. 235-236), une capacité à visualiser et à planifier sa carrière tout au long de sa vie. Il s'agit donc d'aider le consultant à développer cette conscience de carrière et à se projeter dans ses éventuels futurs rôles, cadres et événements de vie. Cela nécessite que le consultant auto-analyse ses intérêts, ses valeurs, ses aptitudes et ses compétences et qu'il prenne la mesure des facteurs de genre, d'origine ethnique, de religion et de statut socio-écono mique qui interviennent dans son propre développement. Dans une phase ulté rieure, il devra mettre en relation ses objectifs et sa situation actuelle, qu'il analysera et intègrera au cours de sa démarche de résolution de problème.

La démarche de conseil à la carrière est présentée dans ses grandes étapes :

- L'étape initiale a pour objectifs l'identification des buts ou problèmes du consultant, la définition et la clarification des relations consultant-conseiller et des rôles impartis à chacun en vue de l'élaboration d'une alliance de travail (Meara, \& Patton, 1994). Au cours de cette première étape, le conseiller oriente son écoute vers les pensées et sentiments intimes du consultant (et vers leur dynamique sous-jacente).

- Le recueil de l'information relative au consultant constitue une étape importante. Les procédures d'évaluation qualitatives 膯 comme l'interview structurée d'évaluation de carrière présentée en annexe de l'article, ou le génogramme de carrière (McGoldrick, \& Gerson, 1990) 區 et quantitatives sont des moyens qui permettront de réunir cette information, de clarifier et de spécifier les objectifs ou les problèmes à considérer.

- La compréhension du comportement $d u$ consultant en termes de carrière et la formulation d'hypothèses. A mesure que s'effectue le recueil d'informations, s'enclenche la phase de compréhension et d'élaboration d'hypothèses. Les consultants ont l'occasion de mettre en lumière leur histoire personnelle et celle de leur groupe de référence. La prise en compte des quatre facteurs que sont le genre, l'origine ethnique, la religion et le statut socio- 
économique, amène les consultants à réfléchir aux influences que ces facteurs ont pu exercer sur leurs représentations d'eux-mêmes, des autres et du monde dans lequel ils vivent. Au cours de cette étape, l'écoute réactive des résistances possibles du consultant est importante.

- La mise en œuvre du counseling consiste à assister le consultant dans la réalisation de ses buts ou la résolution de son problème dans le cadre de l'alliance de travail évoquée plus haut.

- La définition des buts de carrière et des plans d'action. Au cours de cette étape, le conseiller assiste le consultant.

- L'évaluation des résultats et la clôture de la relation.

Gysbers et ses collègues abordent enfin la question de l'efficacité du conseil à la carrière et ils présentent les premiers résultats d'une enquête conduite par une équipe de chercheurs de l'université du Missouri à Columbia.

Le lecteur français aura noté de fortes ressemblances entre la démarche de bilan de compétences (Aubret, 1996; Taïeb \& Blanchard, 1997) et la démarche présentée par Gysbers et ses collègues. Toutefois, dans leur conception de la démarche de conseil à la carrière, la dimension psychologique de la relation conseiller-consultant est beaucoup plus fortement affirmée, Gysbers et ses collègues ne séparant pas le counseling de carrière et le counseling personnel. Selon leur conception, le développement de carrière concerne toute la vie et pas seulement la vie professionnelle et il est donc étroitement lié au développement personnel. Il en résulte que, dans leur optique, le conseil de carrière inclut l'aide à la résolution de difficultés relevant d'un large champ, comme les crises personnelles, le manque d'information sur les filières de formation et le marché du travail, les difficultés relationnelles avec le conjoint, les enfants, les collègues de travail et les supérieurs hiérarchiques.

Dans leur article, Conrad Lecomte et Vincent Guillon traitent de la question des facteurs communs au counseling personnel, au counseling de carrière et à la psychothérapie. Ils développent des positions voisines de celles de Gysbers, Heppner et Johnston, positions qui permettent à leur sens de comprendre un certain nombre d'évolutions, encore limitées, en France même, évolutions qui leur paraissent nécessaires et souhaitables. Pour le moment, toutefois, il y a bien, entre la France et l'Amérique du Nord, des différences de conceptions. Le terme français conseil n'est pas l'équivalent du terme américain counseling. De même, en France, la psychothérapie concerne fondamentalement le champ de la pathologie, alors qu'en Amérique du Nord elle concerne tout autant sinon plus les problèmes ordinaires de changement. En France, la psychologie $\mathrm{du}$ conseil est beaucoup moins développée que la psychologie du counseling en Amérique du Nord, que ce soit sur le plan de l'enseignement universitaire ou des pratiques professionnelles. De plus, la conception française dominante n'in clut pas la thérapie dans la psychologie du conseil en orientation (Angeville \& Bellenger, 1989a, 1989b ; Blanchard, 1996 ; Revuz, 1991 ; Lhotellier, 1996, Zarka dans ce numéro) et les psychologues du conseil ne reçoivent pas une formation de thérapeute. Comme le notent Leong et Blustein $(2000$, p. 8$)$, « il est maintenant tout à fait clair que le counseling s'inscrit dans un contexte culturel. Pour comprendre quels sont les aspects du counseling qui sont communs aux différentes cultures et ceux qui sont spécifiques à telle ou telle culture, nous avons besoin d'entreprendre une étude comparative globale sur les pratiques de counseling dans le monde ».

31 Ce sont donc essentiellement, comme dans l'article précédent, des conceptions très développées en Américaine du Nord, du counseling personnel, du counseling de 
carrière, et de la psychothérapie qui sont présentées dans l'article de Conrad Lecomte et de Vincent Guillon. Ils rappellent d'abord quelques éléments d'histoire de la psychologie du counseling. Dès ses débuts, la psychologie du counseling s'est appuyée sur les ressources, sur les points forts de la personne, en réaction aux conceptions centrées sur la maladie et la pathologie, faisant davantage appel aux travaux de Rogers qu'aux travaux de Freud. C'est en effet avec les travaux théoriques et les pratiques initiées par Rogers au début des années 1940 que la psychologie du counseling disposera d'une conception psychothérapeutique non médicale, non analytique et centrée sur les ressources de la personne. Après la deuxième guerre mondiale, des débats s'engagent sur l'importance et la place à accorder aux activités thérapeutiques, au counseling personnel et au counseling de carrière. Les psychologues américains du counseling en arrivent à définir trois grandes fonctions du counseling : 1) une fonction développementale et éducative, 2) une fonction préventive et 3) une fonction thérapeutique dite remédiative. Toutefois, cela ne permet pas d'éclairer la question de l'articulation entre counseling vocationnel, counseling personnel et psychothérapie.

Beaucoup d'auteurs, nous avons vu que c'est le cas de Gysbers et de ses collègues, insistent sur les liens existant entre counseling de carrière et counseling personnel. En ce qui concerne les facteurs communs au counseling de carrière et à la psychothérapie, Conrad Lecomte et Vincent Guillon mettent en avant l'importance des relations entre travail et santé mentale : répercussion psychologiques de la perte d'emploi, liens entre la satisfaction dans la carrière et les facteurs de dépression et d'anxiété... D'où l'intérêt d'étudier et de conceptualiser dans leur interaction les aspects psychologiques et vocationnels. Les résultats des recherches sur les facteurs communs au counseling de carrière et à la psychothérapie, suggèrent que les trois composantes de la relation (le transfert, le contre-transfert, et surtout l'alliance de travail) entrent pour une part importante dans les effets des démarches d'aide. On notera à ce sujet qu'en matière de counseling et de thérapie, existe une tradition américaine ancienne et active de recherche sur les processus et l'efficacité de la relation d'aide (Hill \& Corbett, 1996), ce qui n'est pas le cas dans le champ de la psychologie clinique en France, même si ces recherches commencent à se développer (Bourguignon \& Bydlowski, 1995 ; DondéMohseni et al., 1988 ; Masse, Blanchet, \& Poitrenaud, 1999 ; Thomassin, 1999). Certaines recherches laissent à penser que la relation d'aide est aussi importante en counseling de carrière qu'en thérapie. Le développement, par des psychanalystes américains comme Bordin, de théories sur la relation d'objet, le soi et le moi, a fourni un cadre permettant d'intégrer les problèmes vocationnels et non vocationnels. Signalons, pour la petite histoire, qu'une présentation en français du livre de Bordin, Psychological counseling, paru en 1955, a été faite dans le Bulletin de Psychologie par D. Super (1959, pp. 531-536).

Conrad Lecomte et Vincent Guillon défendent une approche intégrée de la pratique du counseling de carrière et de la psychothérapie dans la mesure où :

- intervenir auprès d'une personne, c'est aborder et toucher l'organisation de toute l'expérience subjective ;

- le changement a un caractère pluridimensionnel ;

- l'efficacité de toute forme d'intervention dépend de la qualité de la relation et en particulier de la qualité de l'alliance de travail ;

- toute forme d'intervention s'appuie sur la reconnaissance de l'expérience subjective unique du sujet et de son contexte de vie. 
Toutefois, counseling de carrière et psychothérapie ont également des spécificités. Ainsi, lorsque le processus d'aller-retour entre enjeux vocationnels et personnels est compromis parce que des conflits intrapsychiques ou interpersonnels prennent une place prépondérante, un travail thérapeutique à long terme peut alors s'imposer. Cette conception intégrative des aspects vocationnels, personnels et thérapeutiques nécessite évidemment que les conseillers soient formés au counseling de carrière et à la psychothérapie, ce qui est le cas aux Etats-Unis et au Québec mais pas en France.

C'est sur les limites du conseil que Josette Zarka porte sa réflexion dans son article. Depuis plusieurs années, Josette Zarka travaille sur ce thème et contribue ainsi à un travail d'explicitation des types de difficulté rencontrés par des conseillers d'orientation-psychologues au cours d'entretiens de conseil. L'article qu'elle a écrit pour ce numéro s'appuie sur des cas qui lui ont été présentés il y a quelques années par des conseillers d'orientation-psychologues du centre d'application de l' I.N.E.T.O.P. Elle cherche à repérer certaines caractéristiques des situations dans lesquelles le conseil se révèle inopérant. Elle s'efforce également de clarifier ce qui différencie, de son point de vue, le conseil en orientation et la psychothérapie.

Dans son introduction, Josette Zarka distingue :

- le motif de la demande de conseil, c'est-à-dire la ou les raisons de la venue du consultant (par exemple, il va être orienté en B.E.P.) ;

- la demande, appréhendable à travers la façon dont le consultant formule sa ou ses questions en fonction du motif de sa venue ;

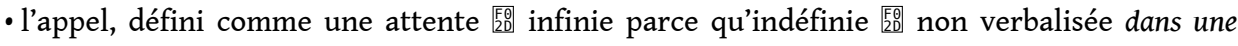
demande.

La première partie traite des situations limites et du conseil interminable. Ces situations se caractérisent par le fait que le consultant utilise le cadre du conseil à contre-courant. L'activité de conseil se dilue car s'il y a de bons motifs, il n'y a pas de demande et l'appel reste indifférencié. Les consultants émettent en fait une demande paradoxale empêchant toute issue, demande du type : «aidez-moi/ne m'aidez pas ».

La deuxième partie traite de la question du conseil bref. L'institution offre une structure d'accueil (dans les cas évoqués, il s'agit de la permanence d'accueil d'un centre d'information et d'orientation) qui structure d'une certaine manière l'interaction de conseil. Cette structure est, d'une part, matérielle : c'est l'espace de la permanence d'accueil qui est organisé d'une certaine manière, lieu soumis à une pression temporelle dans la mesure où il $\mathrm{y}$ a d'autres consultants qui attendent. Cette structure est, d'autre part, psychologique: il y a mise en forme de l'interaction de conseil à travers l'activation de "mécanismes de dégagement », permis par la levée des défenses. "Les opérations défensives s'efforcent de réduire les tensions selon le principe de déplaisir tandis que les opérations de dégagement du moi ont une structure logique, font appel à l'intelligence et accroissent la liberté du sujet en favorisant la mobilité de sa conscience, en le rendant capable de changer de système de référence " (Doron \& Parot, 1991, p. 179). Pour faire entrer le consultant dans cette dynamique de dégagement, les conseillers font jouer quatre facteurs : ils placent, calment, analysent et activent. En plaçant spatialement le consultant, on lui notifie métaphoriquement que l'on tente de le positionner à la fois dans la relation et par rapport à son problème dont on recherche l'assise. Analyse et activation sont les modalités par excellence du dégagement. Trois types de conseil sont évoqués : le conseil informateur, le conseil recadrant et le conseil confrontatif. L'interaction brève à l'accueil est donc une 
interaction de dégagement. Du fait de sa structure, le conseiller n'y reçoit pas ou peu de demandes paradoxales, condamnées d'avance dans la mesure où les limites (espace et temps) créent des ouvertures alors que, dans le conseil interminable, l'absence de limites enferme le consultant dans son problème.

Dans la troisième partie, Josette Zarka aborde la question de la demande ambiguë. Alors que dans la demande paradoxale il y a une absence de demande, dans une demande ambiguë la demande est équivoque ou à double sens. Dans l'ambiguïté il n'y a pas d'appel. En revanche, il y a toujours un écart entre un motif et une demande sousjacente. Si, dans certains cas, le motif fait écran à une demande latente, dans d'autres cas le motif est incompatible avec certaines demandes voilées. Selon Josette Zarka, l'entretien de conseil est le lieu par excellence de l'élaboration, de la formulation et de la structuration d'une demande (Desbuquois \& Gelpe, 1997). Il s'insère dans un dispositif institué où la demande est co-construite [Grossen (1992), quant à elle, présente la demande comme le résultat d'une négociation]

Les entretiens de conseil et les entretiens psychothérapeutiques diffèrent sur deux plans: celui des modalités d'écoute et de compréhension du problème et celui des relations instaurées entre le conseiller et le consultant.

41 En ce qui concerne les modalités d'écoute et de compréhension du problème, une écoute à la fois Inconditionnelle et orientée ou sélective est indispensable, genre d'écoute éminemment active qui peut être structurante pour le consultant. On ne saurait s'interdire aucune interprétation mais doit-on la communiquer? Si la compréhension/ interprétation renvoie directement aux motifs de la consultation, elle fait partie intégrante de l'élucidation de la demande. Mais si l'interprétation s'assortit d'explications éloignées du motif, elle risque soit de renforcer les défenses du consultant, soit de susciter des attentes thérapeutiques et créer l'illusion que l'on pourra y répondre.

42 En ce qui concerne les relations instaurées entre le conseiller et le consultant, le regard du conseiller porte sur le problème que la personne véhicule et qu'ils doivent résoudre ensemble et non pas sur la personne elle-même du consultant comme c'est le cas dans une approche thérapeutique. Il en résulte que le conseil porte sur des conduites et qu'il implique le changement d'une situation donnée et non pas un changement de la personne ni une réduction de ses troubles comme en psychothérapie. Il s'exerce à partir d'une nécessité externe (choix, décision, orientation) qui mobilise les instances du sujet (besoins, désirs, craintes, etc.) à changer. La nature des liens est donc très différente dans le cadre du conseil, où est en jeu une alliance opératoire ou conditionnelle qui porte sur un problème déterminé, et dans le cadre de la thérapie, où est en jeu une alliance inconditionnelle qui porte sur la personne du consultant.

43 Après avoir rappelé que le conseil repose sur un paradoxe fondateur (influencer laisser libre), Josette Zarka conclut son article en soulignant que, dans l'expression « conseil en orientation ", le terme conseil permet au conseiller de se distinguer de l'orienteur, et que le terme orientation permet au conseiller de se démarquer du psychothérapeute.

Pour sa part, Claude Chabrol ne s'intéresse pas aux cas limites mais aux demandes banales des consultants, demandes qui sont aussi les plus fréquentes. Il situe sa réflexion dans une perspective psychosociale et il se propose de clarifier quelques dimensions constitutives des entretiens d'aide inspirés du modèle clinique et d'en tirer quelques conséquences pour les démarches d'orientation. 

reposent de plus en plus sur les mécanismes d'appropriation subjective des normes et des valeurs sociétales, Claude Chabrol fait l'hypothèse que les entretiens d'aide et de conseil tendent à développer implicitement et involontairement cette appropriation «interne» par des techniques psychologiques qui mobilisent un investissement psychique fort de la part du consultant. Or, lorsque le sujet doit faire des choix dans la carrière de vie, scolaire et professionnelle, il va inévitablement se situer par rapport aux croyances, attitudes et valeurs de ses groupes d'appartenance et de référence. Pour diminuer le «biais» de psychologisation, il faudrait sans doute réduire l'usage de l'entretien centré sur le sujet, d'inspiration clinique. Il faudrait plutôt proposer des lieux de parole collectifs afin d'inciter les sujets à une métacognition qui les positionne bien dans leur place sociale et qui leur facilite le repérage des contraintes objectives et subjectives multiples, économiques, sociales, psycho-sociales et cognitives qui surdéterminent leur réflexion et cela pour leur permettre de se construire des stratégies de décision. Pour Claude Chabrol, ce type d'intervention aurait le mérite d'éloigner les sujets de l'illusion du "bon choix ", celui censé correspondre à "ses besoins et désirs profonds ». Notons à ce sujet que le courant de l'ego-écologie a développé une technique d'entretien qui invite le sujet à se focaliser sur ses groupes sociaux d'appartenance (Costalat-Founeau, \& Martinez, 1998 ; ZavalIoni, 1998) afin de l'amener à expliciter les valeurs associées à ces groupes d'appartenance ainsi que son propre positionnement par rapport à ces valeurs. Les lieux de parole collectifs sont proposés par Claude Chabrol en tant que dispositif qui permettrait aux acteurs sociaux, qui se posent la question de leur orientation, de mettre à distance les modes de pensée habituels qui les conduisent finalement à intérioriser les contraintes sociales et à reproduire les « choix » et comportements qui conviennent à leurs positions, c'est à 
dire à celles de la majorité de leur groupe d'appartenance. Il y a là l'idée qu'avant de prendre une certaine distance par rapport aux influences sociales auxquelles nous sommes exposés, il est d'abord indispensable de reconnaître et d'analyser ces influences.

Claude Chabrol souligne que les contrats de communication 医 c'est-à-dire "l'ensemble des croyances mutuelles des interlocuteurs sur les enjeux et les objectifs du dialogue » (Blanchet, 1991, p. 149) 泡 à l'œuvre dans les entretiens cliniques, qu'ils soient rogériens ou psychanalytiques, sont très éloignés des contrats de communication des conversations de la vie sociale courante et que, de plus, ils ne sont probablement pas adéquats aux objectifs du conseil en orientation. Selon lui, l'objectif de l'entretien d'orientation n'est pas à propre ment parler de développer une connaissance de soi, mais plutôt une capacité à analyser les informations sur l'environnement et à réguler ses adhésions groupales en fonction de ses intérêts et de ses possibilités cognitives. Il s'agit également de favoriser l'élaboration d'une expertise cognitive pour calculer les ajustements de toutes les dimensions qui entrent dans une prise de décision d'orientation toujours provisoire, conçue peut-être comme le moins mauvais choix actuellement possible.

Sur le plan des pratiques, Claude Chabrol suggère la mise en place de lieux de parole de formes variées: intra-groupe (familial, de pairs) ou inter-groupes (différenciés par leurs positions de genre : rôles professionnels masculins et fémimins; différenciés par leur âge : cadet et aînés; différenciés par leur statut employeurs et recruteurs, etc.). Dans le domaine de l'éducation à l'orientation, les techniques de groupe (Guichard, 1987 ; Nuoffer, 1987 ; Pelletier et al., 1988 ; Limoges, 1989 ; Sontag, 1996 ; Zerwetz, \& Blanchard, 1998) ont beaucoup été utilisées avec des objectifs variés. Les objectifs visés par Claude Chabrol, à travers ces lieux de parole, sont de présenter et de confronter des points de vue différents et, ainsi, d'enrichir les représentations du monde des participants et de leur permettre de mieux s'ajuster à d'autres milieux que le leur. Ces lieux de parole sont à compléter par des modules d'apprentissage sociocognitifs. En effet, l'orientation nécessite un apport d'informations sur les marchés de la formation et du travail. Il s'agit aussi de favoriser l'apprentissage d'habiletés mentales nécessaires pour traiter les informations sur l'environnement et sur soi, et pour planifier ses activités en vue de la prise de décision. Les entretiens cliniques se voient réservés aux rares cas pour lesquels ils sont utiles, dans la mesure où le conseiller peut disposer d'un temps suffisant.

« En tant que pratique, l'orientation se définit comme une aide apportée à l'individu pour lui permettre de se déterminer. Elle recouvre des activités aux niveaux des individus (information, entretiens, bilans, évaluations, conseil, ...), des groupes (information, animation, bilans, évaluations, conseil, ...) et des institutions (mise en place d'ateliers et de procédures d'aide à l'orientation avec des équipes d'enseignants [Gysbers \& Henderson, 2000] ou de formateurs, mise au point de procédures d'évaluation que l'ensemble des acteurs d'une institution doivent mettre en œuvre, ...). Le principe qui fonde ces actions est celui du développement de l'autonomie de l'individu : c'est la personne concernée qui effectue ses propres choix ; l'aide qui lui est apportée vise à lui permettre de prendre des décisions plus réfléchies » (Guichard et al., 1993, p. 10). Mais dans quels cadres et dans quelles formes identitaires les personnes sont-elles amenées à se décrire ? C'est la question du lien entre les cadres et formes identitaires et les pratiques en orientation que Jean Guichard analyse dans son article. 
Après avoir distingué six grands courants qui ont marqué l'étude du sujet au cours du vingtième siècle (courants personnaliste, psychanalytique, de la psychologie du soi, différentialiste, anthropologique culturaliste ou structuraliste, et psycho-sociaux), Jean Guichard aborde la question de la malléabilité ou de la stabilité de la subjectivité. Selon lui, pour comprendre la dynamique des constructions identitaires, il faut tenir compte à la fois des structures et des interactions.

Jean Guichard s'intéresse d'abord aux liens existant entre les structures du système scolaire et la constitution de certaines formes de la subjectivité des jeunes. Il décrit l'école comme un miroir structuré structurant l'image scolaire de soi des jeunes. La dimension principale qui organise la vision de soi (et des autres) dans ce miroir est celle de l'excellence telle qu'elle est scolairement produite et définie. A l'école, comme l'a montré L. Gottfredson (Guichard, 1993), un adolescent apprend à déterminer la limite supérieure des positions sociales qu'il peut espérer atteindre. De plus, un système scolaire constitue toujours un système de classement des disciplines scolaires allant de pair avec un système de répartition des individus. Ainsi, le système scolaire donne-t-il une forme à l'image de soi des élèves et à leur manière de se projeter dans l'avenir.

Jean Guichard s'intéresse ensuite aux champs sociaux occupés par un adolescent, champs sociaux qui forment un cosmos social, et à l'interaction dialogique qui jouerait un rôle fondamental dans la construction de soi. Il fait l'hypothèse d'une double vicariance des formes identitaires: ces formes identitaires peuvent varier selon le contexte (se présenter comme supporter d'un club de football dans un certain contexte, et comme salarié chez Ford dans un autre contexte) et à l'intérieur d'un même contexte (par exemple, selon la situation, être supporter verbalement violent ou être supporter physiquement violent). Il présente ensuite plusieurs études montrant l'importance des formes identitaires sur les projets d'avenir.

Quelles sont les implications de ces analyses pour les pratiques de conseil en orientation? Elles renvoient à une réflexion sur les fins et les valeurs des pratiques de conseil qui relèvent des techniques de soi. La question fondamentale du conseil est donc celle de ses finalités. Doit-il viser à conduire certains individus à se construire dans quelques formes identitaires aux contours bien définis ou doit-il se proposer de l'aider à s'interroger sur la pertinence des cadres identitaires dans lesquels il se construit et perçoit autrui, questions qui ont à voir avec la conservation ou la transformation de la structure des rap ports sociaux.

La question du conseil amène donc Inévitablement à se poser des questions politiques (Huteau, 1999), philosophiques, et éthiques (Bourguignon, 1994, 2000 Code de déontologie des psychologues, 1997 ; Facy, 1999 ; Huteau, 2000 ; Puel \& Solazzi, 1996 ; Reuchlin, 1971a, 1971b). Elle amène aussi à s'interroger sur les délimitations de son champ d'intervention. Lorsque Alexandre Lhotellier définit le conseil comme: «ni thérapie, ni diagnostic, ni contrôle, ni assistance, ni commerce, ni endoctrinement, ni manipulation, ni subversion ", il précise ce qu'il considère être des dérives du conseil. Christine Revuz (1991), quant à elle, souligne que le conseiller en bilan de compétences n'est ni thérapeute ni expert. Or, les auteurs nord-américains défendent, eux, au contraire, l'idée d'une continuité entre counseling de carrière, counseling personnel et thérapie. La dérive du conseil en orientation la plus souvent évoquée en France porte sur les conséquences possibles de l'individualisation de l'orientation (Demazière, 1995, pp. 77-79), c'est à dire: une psychologisation de problèmes qui sont essentiellement socio-économiques et, plus précisément, une tendance à faire internaliser par les 
consultants que leur situation dépend essentiellement d'eux. Josette Zarka (1986) signale l'importance du cadre institutionnel dans lequel l'activité de conseil s'inscrit. Traqueuse de paradoxes, elle souligne que c'est précisément de son absence de pouvoir institutionnel en matière d'affectation que le conseiller tire son pouvoir de conseil. Si ce principe éthique n'est pas respecté, l'activité de conseil se transforme alors en technique de manipulation.

La variété des sois professionnels actuels ou possibles des conseillers est certainement à lier, pour une bonne part, à leurs choix politiques [Michel Huteau (1999, p. 23) rappelle que jusqu'à la fin des années 1960, beaucoup de conseillers avaient le sentiment de contribuer à une plus grande justice sociale, sentiment qui était une composante de leur identité professionnelle], au cadre institutionnel dans lequel ils travaillent, à leurs options philosophiques, à leurs choix théoriques et techniques mais aussi aux types de demandes de leurs consultants. Nous n'aborderons ici que la question des connaissances théoriques et techniques sur lesquelles les conseillers appuient leurs pratiques et de leurs liens.

Pour les psychologues, selon Le Ny (1999), il y a deux façons de penser la psychologie: la psychologie clinique sous ses formes professionnelles et académiques et la psychologie expérimentale-cognitive. À ces psychologies professionnelles s'ajoutent la psychologie quotidienne de tout un chacun et la psychologie philosophique, déjà évoquée (Fraisse, 1985). Nous avons vu qu'il y a également plusieurs façons de concevoir le conseil en orientation, ses différents courants pouvant être rattachés aux différents types et courants de la psychologie (Angeville \& Bellenger, 1989a, 1989b; Bujold, 1989). C'est aujourd'hui une prétention déplacée, pour un psychologue (quel que soit le courant auquel il appartienne), de vouloir expliquer la grande variété des comportements humains à l'aide d'une théorie unique. On peut d'ailleurs penser que " c'est en renonçant à leur visée totalisatrice, en acceptant de n'être plus la théorie de l'homme, mais le dévoilement de certaines dimensions de son comportement que les sciences humaines pourront, semble-t-il, accéder, en même temps qu'à la modestie, à une véritable maturité " (Bouveresse, 1998, p. 64). En matière de sciences de l'éducation, Avanzini (1995, p.6) note que le passage du singulier (la science de l'éducation à la fin du XIXe siècle) au pluriel (les sciences de l'éducation à l'époque actuelle) « est une reconnaissance du fait que l'étude des pratiques éducatives relève nécessairement de plusieurs points de vue, dont chacun aide à connaître les pratiques éducatives sous un angle déterminé, sans qu'aucun parvienne à en épuiser l'analyse et à rendre compte de leur complexité».

Ce sont peut-être des considérations de ce type qui expliquent, pour une part, que les pratiques des conseillers évoluent vers un certain éclectisme (Zarka, 1987). Aucune théorie n'apportant un ensemble de réponses satisfaisantes face à la diversité des demandes et des situations, les conseillers vont avoir recours au cadre théorique jugé le plus approprié au type de demande du consultant. Ils doivent d'ailleurs se référer à un champ interdisciplinaire incluant, entre autres, la psychologie de l'orientation, les conceptions éducatives en orientation (Boursier, 1989 ; Boy et al., 1999), la construction du projet, le fonctionnement de l' orientation scolaire et universitaire, l' orientation et l'information professionnelle des adultes (Dugué et al., 1999), les aspects sociaux de l'orientation et notamment ses dimensions sexuées (Vouillot, 1999), la relation formation-emploi, les difficultés d'insertion professionnelles et sociale des jeunes, 
l'histoire de l'orientation et son évolution en Europe et dans le monde... (Danvers, 1993).

En ce qui concerne certains courants classiques du conseil en orientation, on peut penser qu'ils se sont mutuellement influencés. Ainsi, par exemple, si les conseillers utilisent des tests ou des questionnaires dans le cadre de la pratique du bilan de compétences, ils font souvent participer le consultant au choix du type d'épreuve, ils utilisent les résultats comme des données à confronter à d'autres types d'informations et ils présentent ces résultats de façon à ce que le consultant puisse bien les comprendre et se les approprier (Blanchard et al., 1999). On peut reconnaître, dans ce type de pratique de bilan, une influence mutuelle du courant diagnostic-pronostic, du courant centré sur le problème de la personne, et du courant éducatif.

La diversité des points de vue théoriques, en matière de conseil en orientation, entraîne évidemment une diversité des techniques utilisées. Dans le seul champ de l'entretien de conseil, un inventaire non exhaustif des types de théories présentant un intérêt pour la conduite des entretiens (Kidd et al., 1995, p. 21) fait état d'une dizaine de théories [méthode centrée sur le client, de Rogers; méthode de l' accompagnement, d'Egan (1987) ; plan en sept points, de Rodger, théorie du développement professionnel, de Super; théorie de l'appariement, de Holland; méthode psychodynamique, de Roe; méthode fondée sur la structure des possibilités éducatives et socio-économiques, de Roberts ; théorie de l'interaction collective, de Law ; théorie de l' apprentissage social, de Krumboltz ; théorie des construits personnels, de Kelly ; analyse transactionnelle, de Berne]. Un autre inventaire (Kidd, 1996), concernant les techniques d'entretien utilisables en conseil de carrière, présente une dizaine de techniques que l'auteur regroupe en fonction de quatre grands modèles: modèle d'appariement, modèle développemental, modèle centré sur la personne, et modèle dirigé vers un but. On pourrait rajouter à cet inventaire l'entretien de counseling d'emploi de Conrad Lecomte (Lecomte, \& Tremblay, 1987; Deleplancque, 1997), l'entretien à partir d'un matériel de cartes de métiers (Garand, 1978 ; Blanchard et al., 1995 ; Volvey, 1995), l'entretien d'aide à la décision, dit de «bilan-inventaire " (Wheeler \& Janis, 1980), l'entretien d'histoire de vie (Francequin, 1995), l'entre tien de génogramme de carrière (Gysbers et al. dans ce numéro, McGoldrick \& Gerson, 1990)...

La question des théories et des techniques du conseil en orientation et de leur lien, mérite aussi d'être examinée parce qu'elle est très liée à celle du professionnalisme des conseillers.

\section{Les liens entre théories et pratiques du conseil : la question du professionnalisme des conseillers}

61 L'activité de conseil en orientation justifie-t-elle une professionnalisation de ceux qui l'exercent? En France, on a répondu par l'affirmative à cette question depuis longtemps, puisque le diplôme de conseiller d'orientation a été créé dès 1931 (Prost, 1996).

62 Est-il possible de préciser la nature des liens entre formation théorique et pratiques professionnelles ? Bourdoncle (1993) a tenté de répondre à cette question dans le cadre d'une réflexion sur la professionnalisation des enseignants. Il nous semble que les réponses qu'il a apportées concernent un large ensemble de professions qui inclut les 
conseillers en orientation. Bourdoncle a focalisé sa réflexion autour des trois questions suivantes.

\section{Quelle est l'importance des savoirs théoriques dans la reconnaissance sociale d'une profession?}

«Les savoirs constituent l'une des caractéristiques centrales des professions. Sans tomber dans l'idéologie actuelle des professions elles-mêmes, qui les mettent en avant pour justifier leurs privilèges et oublier l'organisation sociale et les structures de pouvoir, qui leur sont tout aussi nécessaires, force est de reconnaître que les savoirs sont essentiels... » (Bourdoncle, pp. 95-96). Aux États-Unis, Drapela (1990, pp. 19-20) souligne que «le fait de reconnaître l'activité de conseil comme celle d'une profession socialement légitime a fortement accru le rôle de la théorie dans la formation des praticiens du conseil. Toutes les professions considèrent que de solides connaissances théoriques sont des prérequis essentiels pour une activité efficace dans leurs domaines d'activité respectifs... On peut ajouter qu'un savoir théorique étendu est souvent considéré comme un indice de professionnalisme véritable à la fois par la communauté des conseillers et par le grand public. Toutefois, tous les conseillers n'adhèrent pas à ce point de vue. Pour certains d'entre eux, les connaissances théoriques sont plutôt une entrave qu'une aide à la pratique professionnelle. Quant aux étudiants, ils préfèrent souvent la pratique à la théorie». Alison Fielding donne, dans son article, des explications à cette désaffection pour la théorie.

\section{Quelle est la nature et la fonction du savoir d'expert?}

64 «Un savoir d'expert est un savoir de haut niveau, à caractère systématique (droit, théologie...) ou scientifique (médecine, ingénierie...). C'est aussi un savoir efficace, qui permet d'atteindre le but souhaité... C'est à cause de ce lien fort entre profession et savoir qu'il y a un lien étroit, souligné par Parsons, entre professions et universités : les universités sont les principales institutions de production du savoir.

Le savoir d'expert est régulièrement mis en œuvre pour résoudre des problèmes quotidiens, ce qui suppose la croyance dans la rationalité technique selon laquelle il suffit d'opérer une sélection judicieuse des moyens techniques appropriés aux fins désirées pour atteindre ces fins et donc pour résoudre le problème... Encore faut-il que le problème soit bien posé, la finalité claire et non conflictuelle, le savoir solide et stable. C'est souvent le cas avec ce que Glazer appelle les professions majeures, comme la médecine ou l'ingénierie. Cela l'est beaucoup moins avec les professions mineures, dont les finalités sont confuses ou conflictuelles (enseignement, travail social) ou dont la pratique relève de plusieurs paradigmes concurrents (psychiatrie, enseignement...). Dans ce dernier cas, il n'y a pas de moyen de choisir les solutions adaptées en l'absence d'un savoir consensuel auquel avoir recours...». En ce qui concerne le conseil en orientation, on a vu que les articles de ce numéro présentent des conceptions théoriques et des techniques différentes.

En réalité, « ces situations problématiques échappant à la rationalité technique, que le problème soit mal posé, qu'il y ait conflit de valeur ou que le cas soit unique, sont nombreuses dans toutes les professions, contrairement à ce que postulait Glazer... Ainsi, en médecine par exemple, $80 \%$ des malades reçus par les praticiens présentent 
des configurations de symptômes différentes de celles qui sont décrites dans les livres de médecine. Face à l'imprévu, les professionnels mettraient en œuvre une espèce d'intuition, de talent ou d'art... Cet art de la pratique, Schön le nomme "réflexion en action". Comment la caractériser? C'est une pensée à la fois d'exploration, de construction d'hypothèse et de test qui s'accomplit d'un même mouvement, au cours même de l'action, et non par réflexion après coup, ou en interrompant momentanément l'action. C'est une réflexion en action, ou mieux, un agir réflexif, s'appuyant beaucoup sur le savoir en action, tout ce savoir que nous mettons quotidienne ment en jeu sans pouvoir cependant bien l'expliciter... Cette épistémologie de la pratique révèle une pensée syncrétique, non entièrement consciente et délibérée, mais cependant efficace dans l'instant et bien différente de la rationalité technique, très analytique et entièrement explicite ».

"Cependant, cet art de poser de manière nouvelle les problèmes, de les résoudre en direct et d'improviser, cette réflexion en action est un exercice de l'intelligence, rigoureux à sa manière et de toute façon nécessaire pour permettre la mise en œuvre des savoirs scientifiques et techniques » (Bourdoncle, 1993, PP. 96-97).

Drapela (1990, p. 19) pense que "la théorie est, pour un professionnel, semblable au plan de vol du pilote qui cherche à ne pas perdre sa route Est-ce une conception idéale ou réelle du fonctionnement d'un conseiller? Les observations d'une enquête sur les pratiques de conseillers d'orientation anglais conduites par Kidd, Killeen, Jarvis et Offer (1995, p. 19) suggèrent que " dans leurs entretiens, les conseillers tendent à appliquer des principes théoriques généraux plutôt que des éléments spécifiques». Dans l'ensemble, les résultats de leur recherche conduisent Kidd et ses collègues «à mettre en doute l'idée que l'orientation professionnelle serait une science appliquée ». Il reste toutefois à s'interroger sur le rapport existant entre les savoirs théoriques et les savoirs pratiques et, en particulier, à discuter la conception d'une pratique comme pure application de théories apprises en formation. La réalité semble en effet plus complexe.

\section{Quels sont les processus qui contribuent à la construction d'un savoir d'expert ou savoir en usage?}

Les travaux de Tardif et de ses collègues (1991) éclairent la question de l'appropriation des savoirs théoriques dans le cadre de l'activité pratique : "Pour les enseignants, les savoirs acquis par l'expérience du métier constituent les fondements de leur compétence... Les enseignants ne rejettent pas globalement les autres savoirs (ceux non acquis par l'expérience) : au contraire, ils les incorporent à leur pratique mais en les retraduisant dans les catégories de leur propre discours. En ce sens, la pratique apparaît comme un processus d'apprentissage à travers lequel les enseignants retraduisent leur formation et l'ajustent au métier... Un tel rapport au savoir chez les enseignants invalide tout programme de formation qui chercherait à transmettre des savoirs à appliquer tels quels parce qu'ils seraient scientifiquement établis par des recherches... Cette conception applicationniste du savoir nous semble contradictoire avec une conception professionnelle de l'activité enseignante, pour autant qu'un professionnel, c'est non seulement celui qui maîtrise un corps de savoir formel, mais aussi celui qui l'interprète pour pouvoir l'appliquer à des cas particuliers Tardif, Lessard et Lahaye (1991) refusent d'ailleurs le rôle de simple interprète du savoir produit par d'autres... car il leur semble que cette interprétation, "cette retraduction 
sur le terrain des contingences professionnelles est porteuse de savoirs propres qu'il importe de mieux connaître dans leur émergence et leur structuration" » (cité par Bourdoncle, 1993, p. 106-108).

En ce qui concerne la question de la relation entre la pratique scientifique et la formation professionnelle des psychologues, Hoshmand et Polkinghorne (1992) pensent que la recherche universitaire ne devrait pas négliger l'étude de la connaissance pratique des psychologues praticiens. Ils estiment que les recherches pourraient notamment se donner pour objectifs

- de clarifier les modèles de compréhension que se fabriquent les psychologues au cours de leur pratique et d'examiner le processus de réflexion éclairée du psychologue, en ne se focalisant pas seulement sur le jugement diagnostique ou la prise de décision relative à un résultat spécifique, comme c'est le plus souvent le cas dans les études sur le jugement clinique ;

- d'aider à affiner les cartes conceptuelles des psychologues en conduisant des enquêtes sur leurs pratiques, en cherchant à les lier à la base des connaissances théoriques formelles.

Ces recherches sont encore peu nombreuses car elles sont longues et difficiles à conduire. Nous avons vu que Zarka a fait des enquêtes sur les difficultés rencontrées par des conseillers d'orientation-psychologues au cours d'entretiens, en vue de préciser les limites de l'espace du conseil. En Angleterre, des enquêtes ont également été conduites sur les pratiques de conseillers d'orientation (Kidd et al., 1995). À I'I.N.E.T.O.P., nous avons engagé des enquêtes sur les représenta tions que des conseillers d'orientation-psychologues se font de certaines de leurs pratiques professionnelles. Les entretiens ont été conduits par des conseillers d'orientationpsychologues stagiaires en formation. Il s'agit d'aider les conseillers, interviewés à propos d'une de leur pratique, à expliciter (Vermersch, 1990) les éléments importants de leurs savoir-faire, les courants théoriques auxquels ils se réfèrent et les valeurs qui les guident. Au cours d'un premier entretien, on se centre sur une description précise de la pratique. Ce n'est qu'au cours d'un second entretien qu'on se centre sur l'explicitation des savoir-faire en jeu, des courants théoriques qui inspirent la pratique, des valeurs qui l'orientent, etc. (Acquier et al., à paraître). Ce travail d'analyse des pratiques professionnelles semble avoir été bien apprécié par les conseillers qui se sont engagés dans ce type d'interview, probablement parce que cet exercice d'explicitation leur a permis de dire et, par là, de construire certaines de leurs compétences professionnelles. L'analyse des pratiques de conseil peut ainsi contribuer à la fois à mieux préciser ce que sont ces pratiques et à accroître le professionnalisme des conseillers.

72 On notera qu'en matière d'analyse du travail, la psychologie du travail a développé des méthodes applicables à l'analyse des activités des conseillers, comme la démarche mise en œuvre par Anne Lancry (2000) pour analyser le travail d'écoutants dans une association qui reçoit des appels téléphoniques de candidats au suicide, ou comme l'entretien d'auto-confrontation croisée d'Yves Clot (1999), méthode qui est également applicable en formation. Quant aux méthodes d'analyse des interactions verbales, elles commencent à être appliquées, en France, aux entretiens thérapeutiques (Masse et al, 1999 ; Thomassin, 1999).

73 Pour conclure, les réflexions sur le conseil en orientation renvoient à la fois à des questions politiques et économiques, à des conceptions philosophiques de l'homme et de la société, et à des systèmes de valeurs qui en orientent les objectifs. C'est pourquoi 
le champ de l'orientation suscite tant de débats, souvent vifs parce que passionnés. Les pratiques de conseil ne peuvent s'appuyer que sur des savoirs scientifiques aux pouvoirs explicatifs limités et sur des techniques dont la validité n'est jamais absolue, mais ce sont précisément ces types de savoirs et les interrogations qu'ils ne cessent d'engendrer qui permettent de différencier les conseillers en orientation des voyants extra-lucides et d'augmenter l'efficacité de leurs actions. Le développement du professionnalisme des conseillers en orientation est d'autant plus important que leur tâche est complexe et que leur action est limitée dans le temps. Ce numéro spécial a pour objectif de présenter une réflexion sur la question du conseil ainsi que différentes conceptions théoriques et pratiques. Nous remercions les auteurs pour leurs contributions et, de façon plus générale, pour leur apport à la psychologie du conseil qui reste encore à développer en France. Nous espérons que les praticiens liront ces articles avec intérêt. Pour finir, nous serions tentés de leur suggérer, face aux difficultés qu'ils rencontrent au cours de leurs pratiques de conseil en orientation, de toujours garder à l'esprit la belle formule de Mark Twain: « Ils ne savaient pas que c'était impossible... alors, ils l'ont fait ». Mais, en les engageant dans ce genre d'exercice spirituel, fréquemment pratiqué à l'époque de Marc-Aurèle, nous leur proposerions une orientation stoïcienne qu'ils ne partagent probablement pas tous. Enfin, cette suggestion serait-elle bien utile, dans la mesure où, comme nous le rappelle La Rochefoucault dans ses Maximes (502), « on donne des conseils, mais on ne donne point la sagesse d'en profiter $"$.

\section{BIBLIOGRAPHIE}

Acquier, S., Blanchard, S., Guillon, V., Ospital, N., Pellet, F., Pierson, E., \& Pietrzyck, C. (à paraître). Pratiques professionnelles de conseillers d'orientation-psychologues et courants du conseil.

Questions d'Orientation.

Angeville, H., \& Bellenger, J. (1989). Quelques courants de la pratique du conseil. Bulletin de l'A.C.O. F, Numéro Spécial «L'entretien en orientation »,14-41.

Angeville, H., \& Bellenger, J. (1989). Réflexions sur une expérience de formation au conseil en orientation. L'Orientation Scolaire et Professionnelle, 18, 2, 111-125.

Aubret, J. (1996). Bilan de compétences, orientation des adultes et trajectoires de vie. Spirale, 18, 27-38.

Avanzini, G. (1995). Des sciences de l'éducation. Cahiers Binet-Simon, 645, 4, 5-26.

Blanchard, S., Volvey, C., Homps, F., \& Prieur, A. (1995). Une technique d'explicitation des intérêts : l'entretien A.D.V.P. L'Orientation Scolaire et Professionnelle, 24, 4, 425-442.

Blanchard, S. (1996). Introduction à l'article de C. Hill et M. Corbett. L'Orientation Scolaire et Professionnelle, 25, 2, 211-216.

Blanchard, S., Sontag, J.-C., \& Leskow, S. (1999). L'utilisation d'épreuves conatives dans le cadre du bilan de compétences. L'orientation Scolaire et Professionnelle, 28, 2, 275-297. 
Blanchet, A. (1991). Dire et faire dire. L'entretien. Paris : A. Colin.

Bourdoncle, R. (1993). La professionnalisation des enseignants : les limites d'un mythe. Revue Française de Pédagogie, 105, 83-119.

Bourguignon, O. (1994). Éthique, déontologie et clinique. L'Orientation Scolaire et Professionnelle, 23, 1, 79-84.

Bourguignon, O. (Dir.), (2000). Numéro spécial « Éthique en psychologie et déontologie des psychologues. Bulletin de Psychologie, tome 53 (1)/445.

Bourguignon, O., \& Bydlowski, M. (Dir.), (1995). La recherche clinique en psychopathologie. Perspectives critiques. Paris : P.U.F.

Boursier, S. (1989). L'Orientation éducative des adultes. Paris : Éd. Entente.

Boutinet, J.-P. (1998). Pertinence et impertinence du projet en orientation. Cahiers Binet-Simon, $656 / 657,3 / 4,11-22$.

Boy T., Cartier, J.-P., Pépin, P.Y., Guichard, J., Fluteau, M., Guillon, V., \& Barbot, A. (1999). Analyse des méthodes éducatives en orientation. L'orientation Scolaire et Professionnelle, 28, 2, 157-223.

Bujold, C. (1989). Choix professionnel et développement de carrière : théories et recherches. Boucherville, Québec, Canada: G. Morin.

Castra, D. (1996). Un point de vue socio-cognitif sur les pratiques de bilan. Carriérologie, 6, 2, 37-44.

Clot, Y. (1999). La fonction psychologique du travail. Paris : P.U.F.

Code de déontologie des psychologues (1997). Pratiques Psychologiques, 3, 71-80

Costalat-Founeau, A.-M., \& Martinez, N. (1998). Identité et changement : comparaison de deux situations de réussite d'adolescentes d'origine culturelle différenciée. L'Orientation Scolaire et Professionnelle, 27, 1, 23-46.

Danvers, F. (1993). L'orientation des jeunes et des adultes : vers la constitution d'un champ interdisciplinaire. Repères Bibliographiques, 30, 109-136.

Deleplancque, B. (1997). Le counselling d'emploi. Démarche et pratique en orientation professionnelle. Lomme (59) : A.F.P.A./I.N.I.O.P.

Demazière, D. (1995). La sociologie du chômage. Paris : La Découverte.

Demazière, D. (1999). Les paradoxes des chômeurs de longue durée. In E. Dugué, R. Guerrier, L. Le Bars, C. Lespessailles, M. Maillebouis, \& C. Mathey-Pierre (Dir.), L'orientation professionnelle des adultes. Contributions de la recherche, état des pratiques, étude bibliographique, (pp. 233-238). Afpa/ Centre Inffo/Cnam. Collection Études no 73. Marseille : C.E.R.E.Q.

Desbuquois, J., \& Gelpe, D. (1997). L'entretien psychologique d'analyse initiale de la demande. Document interne (79 p.). Lomme (59) : A.F.P.A./I.N.I.O.P.

Divay, S. (1998). La recherche d'emploi protocolaire ou de nouvelles pratiques de conseil sur le marché du travail. Groupe de Recherche Innovations et Sociétés (G.R.I.S., Département de Sociologie, Université de Rouen), 3, 4-42.

Dondé-Mohseni, S., Vila, G., \& Mouren-Simeoni, M.-C. (1998). La thérapie cognitive de l'adolescent déprimé. L'Orientation Scolaire et Professionnelle, 27, 4, 545-566.

Doron, R., \& Parot, F. (1991). Dictionnaire de psychologie. Paris : P.U.F.

Drapela, V.J. (1990). The value of theories for counseling practitioners. International Journal for the Advancement of counselling, 13, 16-26. 
Drolet, J.-L (1995). L'insertion socio-professionnelle en tant que relation intentionnelle au monde. Carriérologie, 6, 1, 117-140.

Dubois, N. (1996). Internalité et évaluation. Carriérologie, 6, 2, 145-159.

Dumora, B. (1997). L'évolution des projets d'orientation. Carriérologie, 6, 25-34.

Dugué, E, Guerrier, R., Le Bars, L., Lespessailles, C., Maillebouis, M., \& Mathey-Pierre, C. (Dir.). Afpa/Centre Inffo/Cnam (1999). L'orientation professionnelle des adultes. Contributions de la recherche, état des pratiques, étude bibliographique. Afpa/Centre Inffo/Cnam. Collection Études no 73. Marseille : C.E.R.E.Q.

Egan, G. (1987). Communication dans la relation d'aide. Laval, Québec : Editions Etudes Vivantes.

Facy, H. (1999). Responsabilité professionnelle et droit des usagers. Questions d'Orientation, 1, 103-111.

Fraisse, P. (1985). Il y a trois psychologies. In La communication. Symposium de I'A.P.S.L.F., Montréal, 1983. Paris : P.U.F.

Francequin, G. (1995). Histoire de vie et pratiques de l'orientation. L'Orientation Scolaire et Professionnelle, 24, 2, 301-325.

Gangloff, B. (1999). Le bilan de compétences : une utopie nécessaire à l'anesthésie sociale. Psychologie et Psychométrie, vol. 20, 4.

Garand, M. (1978). De l'orientation à l'activation. L'Orientation Scolaire et Professionnelle, 7, 4, 299-324.

Grossen, M. (1992). Intersubjectivité et négociation de la demande dans un entretien thérapeutique. In A.-M. Perret-Clermont (Dire), L'espace thérapeutique (pp. 165-191). Neuchâtel : Delachaux \& Nestlé.

Guichard, J. (1987). Les D.A.P.P. : nouvelle méthode pour aider les lycéens et les étudiants à construire leurs projets. L'Orientation Scolaire et Professionnelle, 16, 4, 347-355.

Guichard, J. (1993). L'école et les représentations d'avenir des adolescents. Paris : P.U.F.

Guichard, J. (1997). Changements sociaux et pratiques d'orientation : analyse de la notion d'éducation à l'orientation. Questions d'Orientation, 4, 11-37.

Guichard, J., Forner, Y., \& Danvers, F. (1993). Les services d'orientation scolaire et professionnelle en France. Contribution à l'étude coordonnée par A.G. Watts, J. Guichard, P. Plant, \& L. Rodriguez pour la Commission des Communautés européennes.

Guichard, J., \& Huteau, M. (1997). L'école et les intentions d'avenir professionnelles des adolescents. In H. Rodriguez-Tomé, S. Jackson, \& F. Bariaud (Dir.), Regards actuels sur l'adolescence (pp. 207-234). Paris: P.U.F.

Gysbers, N.C., \& Henderson, P. (2000, 3rd ed.). Developing and managing your school guidance program. Alexandria, V.A.: American Counseling Association.

Hadot, P. (1997, 2c éd.). La citadelle intérieure. Introduction aux pensées de Marc Aurèle. Paris : Fayard.

Hill, C.E., \& Corbett, M.M. (1993/trad. 1996). La recherche sur les processus et l'efficacité de la relation d'aide en psychologie du conseil : Histoire et perspectives. L'Orientation Scolaire et Professionnelle, 25, 2, 217-265.

Hoshmand, L.T. \& Polkinghorne, D.E. (1992). Redefining the science practice relationship and professionnal training. American Psychologist, 47, 1, 55-66. 
Hurbin, J. (1997). L'orientation professionnelle à E.D.F.-G.D.F. : une fonction en voie de légitimation. In L'orientation face aux mutations du travail (pp. 173-178). La Découverte \& Syros/ Cité des Sciences et de l'Industrie.

Huteau, M. (1996). Les problématiques de l'orientation. Actes du séminaire « Pour le développement de la culture de l'orientation » (pp. 13-22). Académie de Créteil, 5-6 octobre 1995.

Huteau, M. (1999). Psychologie et société : l'évolution du rôle et des méthodes des conseillers d'orientation au cours des années 1920 à aujourd'hui. Questions d'Orientation, l, 13-24.

Huteau, M. (2000). Ethique et pratiques de l'orientation en milieu scolaire. Bulletin de Psychologie, Numéro spécial « Éthique et déontologie », tome 53 (1)/445, 115-121.

Kidd, J.M., Killeen, J., Jarvis, J., \& Offer, M. (1995), L'orientation professionnelle est-elle une science appliquée ? Rôle de la théorie dans la consultation d'orientation professionnelle en Grande-Bretagne. L'Orientation Scolaire et Professionnelle, 24, 1, 19-37.

Kidd, J.M. (1996). The career counseling interview. In A.G. Watts, B. Law, J. Killeen, J.M. Kidd, \& R. Hawthorn, Rethinking careers education and guidance. Theory, policy and practice (pp. 189-209). London: Routledge.

Kihlstrom, J.F., Cantor, N., Sumi Albright, J., Chew, B.R., Klein, s.B., \& Nedenthal, P.M. (1988/1992). Traitement de l'information et étude du soi. In M. Piolat, M.-C. Hurtig, \& M.F. Pichevin. (1992). Le soi. Recherches dans le champ de la cognition sociale (pp. 205-247). Lausanne : Delachaux \& Niestlé.

Lagache, D. (1962, 5e éd.). La psychanalyse. Que-sais-je ? Paris: P.U.F.

Lancry, A. (2000). L'analyse du travail dans certains emplois de service aux personnes. Séminaire de I'I.N.E.T.O.P./C.N.A.M., Paris, 28 janvier 2000.

Lecomte, C, \& Tremblay, L. (1987). Entrevue d'évaluation en counselling d'emploi. Montréal, Québec : Institut de Recherches Psychologiques.

Lemaire, J.G. (1986). Le couple, sa vie, sa mort. Paris: Payot.

Leong, F.T.L., \& Blustein, D.L. (2000). Toward a global vision of counseling psychology. The Counseling Psychologist, vol. 28, 1, 5-9.

Le Ny, J.-F. (1999). La psychologie est durablement duale. Bulletin de Psychologie, 52 (2), 440, 273-285.

Lhotellier, A. (1996). La démarche de consultance et la formation des conseillers. Spirale, 18, 139-160.

Lhotellier, A. (1997). La grande illusion du projet préfabriqué. Carriérologie, vol. 6, 3/4, 105-115. Limoges, J. (1989). L'orientation et les groupes dans une optique carriérologique. Québec : Université de Sherbrooke.

Loss, I. \& Parlier, M. (Dir.), (1996). Mobilité et orientation professionnelles dans les organisations. L'Orientation Scolaire et Professionnelle, 25, numéro spécial.

Masse, L., Blanchet, A, \& Poitrenaud, S. (1999). Construction des univers référentiels en psychothérapie. Psychologie Française, 44, 4, 349-360.

Mathey-Pierre, C. (1999). Quelle orientation pour les jeunes demandeurs d'emploi ? In E. Dugué, R. Guerrier, L. Le Bars, C. Lespessailles, M. Maillebouis, \& C. Mathey-Pierre (Dir.), L'orientation professionnelle des adultes. Contributions de la recherche, état des pratiques, étude bibliographique (pp. 243-251). Afpa/Centre Inffo/Cnam. Collection Études no 73. Marseille : C.E.R.E.Q. 
Meara, N., \& Patton, M. (1994). Contribution of the working alliance in the practice of career counseling. The Career Development Quartely, 43, 161-177.

McGoldrick, M., \& Gerson, R. (1990). Génogrammes et entretien familial. Paris : E.S.F.

Nuoffer, J. (1987). L'atelier d'orientation. Association suisse pour l'orientation scolaire et professionnelle : Zurich \& Lausanne.

Pegeault, M., Ber-Schiavetta, A. (1997). Une réponse associative fondée sur l'éthique : l'autoorientation. In L'orientation face aux mutations du travail (pp. 179-183). La Découverte \& Syros/Cité des Sciences et de l'Industrie.

Pelletier, D., Noiseux, G., \& Pellerano, J. (1988). Éducation des choix (livrets pour les niveaux 6e, 5e, 4 e et $3 e$ et guide de l'accompagnateur). Issy-les-Moulineaux : E.A.P. Puel, H., \& Solazzi, R. (1996). Éthique et orientation. Spirale, 18, 129-138.

Prost, A. (1996). Des professions à l'école : jalons pour une histoire de l'orientation en France. Vie Sociale, 5, 11-24.

Reuchlin, M. (197 la). Limites et portée d'une déontologie professionnelle. In M. Reuchlin (Dir.), Traité de psychologie appliquée, tome I (pp. 231-238). Paris : P.U.F.

Reuchlin, M. (197 lb). Rôle et responsabilités propres du conseiller d'orientation scolaire et professionnelle. B.I.N.O.P., 27, 3-20.

Revuz, C. (1991). Ni thérapeute ni expert. L'entretien de bilan-orientation à la recherche de sa spécificité. Éducation Permanente, 108, 57-76.

Rey, A. (1995). Dictionnaire historique de la langue française. Paris : Ed. Le Robert.

Shaftesbury, A. (1710/1993). Exercices. Paris : Aubier.

Sontag, J.-C. (1996). Une aide à la prise de décision : l'exemple d'un programme d'intervention de C.I.O. en classe de terminale. L'Orientation Scolaire et Professionnelle, 25, 1, 97-127.

Sullerot, E. (1996). L'orientation professionnelle des adultes : l'expérience de « retravailler». Spirale, 18, 9-26.

Super, D.E. (1959). Les techniques du conseil et l'analyse des interviews. Bulletin de Psychologie, tome XII/10, 162, 524-531

Taïeb, D., \& Blanchard, S. (1997). Le bilan de compétences : une démarche d'aide à la décision de carrière. Connexions, 70, 79-93.

Tardif, M., Lessard, C., \& Lahaye, L. (1991). Les enseignants des ordres d'enseignement primaire et secondaire face aux savoirs. Esquisse d'une problématique du savoir enseignant. Sociologie et Sociétés, 23, 1, 55-69.

Thomassin, P. (1999). Déconstruction et reconstruction de l'identité narrative au cours d'une psychothérapie. Psychologie Française, 44, 4, 371-381.

Tourette-Turgis, C. (1996). Le counseling. Que sais-je ? Paris: P.U.F.

Vermersch, P. (1990). Questionner l'action : l'entretien d'explicitation. In A. Blanchet (Dir.), Anatomie de l'entretien. Psychologie Française, 35, 3, 227-235.

Volvey, C. (1995). Pratique de l'entretien A.D.V-P. L'Orientation Scolaire et Professionnelle, 24, 4, 443-462.

Vouillot F. (Dir.), (1999). Filles et garçons à l'école : une égalité à construire. Collection Autrement Dit. Paris : C.N.D.P. 
Wheeler, D., \& Janis, L. (1980). A practical guide for making decisions. New York : The Free Press.

Zarka, J. (1986). Essai de modélisation de la pratique de conseil en orientation. Texte dactylographié. Paris : Bibliothèque de I'I.N.ET.O.P.

Zarka, J. (1987). Éclectisme ou pluralisme. Bulletin de l'Acof, 315, 30-52.

Zavalloni, M. (1998). Vers une orientation et une intervention interactive : l'identité comme hypertexte (imis). L'Orientation Scolaire et Professionnelle, 27, 1, 5-22.

Zerwetz, M., \& Blanchard, S. (1998). L'émergence des conceptions éducatives de l'orientation en France : quelques jalons historiques. Questions d'Orientation, 3, 27-70.

\section{AUTEUR}

\section{SERGE BLANCHARD}

Chercheur au service de recherche de I'INETOP

Service de Recherche, INETOP-CNAM, 41, rue Gay-Lussac, 75005 Paris. Courrier électronique :

blanchard@cnam.fr 